\title{
Prevalência de sobrepeso e obesi dade e fatores associados em adolescentes na região centro-oeste do estado de São Paulo (SP, Brasil)
}

\author{
Prevalence of overweight and obesity, and associated factors \\ in adolescents, at the central West area of the State São Paulo \\ (SP, Brazil)
}

\author{
Sílvia H elena de Carvalho Sales-Peres ${ }^{1}$ \\ Suzana Goya ${ }^{1}$ \\ Rute M oreira de Freitas Sant'Anna ${ }^{1}$ \\ Henrique M endes Silva ${ }^{1}$ \\ Andréde Carvalho Sales-Peres ${ }^{1}$ \\ Ricardo Pianta Rodrigues da Silva ${ }^{1}$ \\ José Roberto Pereira Lauris ${ }^{1}$ \\ José Roberto de $M$ agalhães Bastos ${ }^{1}$
}

\footnotetext{
${ }^{1}$ Departamento de Odontopediatria Ortodontia eSaúde Coletiva daFaculdade de Odontologia deBauru, Universidade de São Paulo. Alameda O ctávio Pinheiro Brisolla 9-75. 17012-901 Bauru SP.shcperes@usp.br
}

Abstract The aim of the study was to evaluate the relationship between the Body M ass Index (BM I) and the DM FT index, in 207 adolescents aged 12 yearsold, from 8 public and private schools of the central west area of São Paulo State. From a sample of 38012 year-old adolescents, both genders, 207 were examined. We used the index DM FT, CBI for weight, measured of stature and applied a questionnaire about alimentary habits, characteristic anthropometrics and physical activity. Regarding body weight, $55.93 \%$ was normal, $35.59 \%$ had low weight, and $8.47 \%$ were pre- obese in private schools. In the public schools, $52.03 \%$ had normal weight, $41.22 \%$ had low weight, $4.73 \%$ were pre-obese and $2.03 \%$ were obese, without significant difference $(p=0.45)$. The DMFT of public schools was 2.16, compared to 0.23 in private schools $(p<0.05)$, with $39.2 \%$ of caries-free individuals in public schools and $88.1 \%$ in private schools. There was no correlation between the increase in BMI and the increase in DM FT. There was negative correlation between socioeconomic conditions and dental caries. It was concluded that, even though the pre- obese and obesegroups presented a higher frequency of food ingestion, obesity was not correlated with the increase in dental caries. H owever, the socioeconomic conditions were determinant for this occurrence.

Key words Obesity, Adolescent, Dental caries, Epidemiology, Body mass index
Resumo Objetivou-se avaliar a relação entre 0 ÍndicedeM assa Corporal (IM C) eo índice CPOD em 207 adolescentes de 12 anos, de 8 escolas públicas e particulares da região centro- oeste do estado de São Paulo. A amostra foi constituída por 380 adolescentesaos 12 anos, deambos os gêneros, sendo examinados 207. U tilizou-se o índice CPOD, IM C para peso, medida de estatura, e aplicou-se questionário sobre hábitos alimentares, características antropométricas e atividade física. Quanto ao peso corpóreo, 55,93\% apresentaram normal (G4), 35,59\% de baixo peso (G3) , 8,47\% de préobesos (G2), nas escolas particulares. Nas públicas, 52,03\% apresentaram normal, baixo peso $41,22 \%$, pré-obesos $4,73 \%$ e obesos (G1) $2,03 \%$; não houve diferença significativa $(p=0,45)$. Verificou-sequeo CPOD nas escolas públicasfoi 2,16 e nas particulares, $0,23(p<0,05)$, sendo que $39,2 \%$ das crianças estavam livres de cárie nas municipais enas particulares, 88,1\%. Não houve correlação do maior IMC com o incremento de CPOD. H ouve correlação negativa entre condições socioeconômicas e índice de cárie dentária. Concluiuse que os grupos pré obesos e obesos, embora houvesse maior frequência de ingestão de alimentos, não apresentaram correlação com o incremento de cárie dentária, mas as condições socioeconômicas foram determinantes para essa ocorrência. Palavras-chave Obesidade, Adolescente, Cárie dentária, Epidemiologia, ÍndicedeM assa Corporal 
Introdução

A situação nutricional da população é essencial para aferir a evolução das condições de saúde e de vida, considerando seu caráter multicausal, relacionado ao grau de atendimento das necessidades básicas. 0 papel da dieta no desenvolvimento da cárie dentária já está bem estabelecido. No entanto, a avaliação da prevalência do sobre peso e obesidade e os respectivos fatores associados têm sido pouco estudados.

Há uma tendência de ascensão das prevalências de sobrepeso e de obesidade, tanto nos países desenvolvidos quanto naqueles em desenvolvimento. Aspectos singulares da transição nutricional ocorrida neste século são encontrados em cada país e região do mundo, contudo são elementos comuns: 0 declínio no dispêndio energé tico, uma dieta rica em gordura (particularmente as de origem animal), açúcar e alimentos refinados, porém reduzida em carboidratos complexos e fibras ${ }^{1}$.

A presença da desnutrição, deficiência demicronutrientes, excesso de peso e outras doenças crônicas não transmissíveis coexistindo nas mesmas comunidades e, muitas vezes no mesmo domicílio, caracteriza a transição nutricional ${ }^{2}$. 0 padrão de transição epidemiológica coexiste em países onde predominam óbitos por doenças cardiovasculares, a prevalência de obesidadeéalta, enquanto em países com elevadas taxas de desnutrição predominam os óbitos por doenças infecciosas ${ }^{3}$. Essefenômeno étraduzido em um dos maiores desafios para as políticas públicas no momento e exige um modelo de atenção à saúde pautado na integralidade do indivíduo com uma abordagem centrada na promoção da saúded. As doenças crônicas não transmissíveis são as que mais demandam ações, procedimentos e serviços de saúde, proporcionando uma sobrecarga no serviço público.

M oraes et al. ${ }^{5}$ analisaram diferentes estudos nos quais foi observada a tendência de aumento da obesidade em áreas urbanas de países pobres. Ela estava relacionada, principalmente, ao incremento da renda, em contraste como os países situados em níveis mais avançados de desenvolvimento, onde 0 aumento da renda resultou em tendência de declínio da obesidade.

$\mathrm{Na}$ Pesquisa de Orçamentos Familiares (POF) de2002-2003, verificou-seuma prevalência de baixo peso de 2,8\% e 5,4\% entrehomense mulheres, respectivamente. Ressalta-se que, segundo a $\mathrm{OM} \mathrm{S}^{6}$, prevalência de bai xo peso até $5 \%$ é considerada aceitável para países em desenvol- vimento. Entre os adolescentes, os resultados demonstraram frequências relativamente baixas de déficits ponderais, alcançando $3,7 \%$.

0 outro lado da transição nutricional da população brasileira refere-se às prevalências crescentes do excesso de peso e de obesidade. Como consequência dos novos e piores hábitos alimentares, os brasileiros engordaram ao longo das últimas três décadas ${ }^{7}$. Os inquéritos populacionais têm mostrado um aumento expressivo do problema entre os homens e as mulheres nas diferentes regiões brasileiras, alcançando $41 \%$ de excesso de peso e 8,8\% de obesidade 4 .

Nas famílias brasileiras, de todas as classes e de todas as regiões, persiste 0 alto consumo de açúcar, principalmente de refrigerantes, sendo baixo 0 consumo de frutas e hortaliças, mas alto o de gordura. 0 problema do excesso de peso não é mais exclusividade das pessoas com renda mais elevada; há mais gordos que magros na população de baixa renda. Entre os $20 \%$ dos indivíduos mais pobres do país, $27 \%$ dos homens estão com peso acima do adequado e 9,5\% com falta de peso. Por sua vez, entre as mulheres de baixa renda, 38,2\% estão com excesso de peso e $6,6 \%$ com peso inferior ao recomendado.

$\mathrm{Na}$ população adulta, a obesidade aumenta o risco de adoecer e de morrer, e nas crianças vêm se convertendo em um problema que terá profundas repercussões em Saúde Pública. A obesidade infantil éum tema que, recentemente, vem ganhando destaque, tanto em países desenvolvidos como em desenvolvimento, pois as crianças que apresentaram sobrepeso poderão tornar-se adultas com excesso de peso.

Dentro do processo de transição nutricional, pode-se destacar também a possível correção do déficit estatural. 0 emprego do exame antropométrico na avaliação do estado nutricional de indivíduos é amplamente recomendado pelos organismosinternacionaisenacionais de saúde, por ser prático e de menor custo ${ }^{9}$.

0 Índice de M assa Corporal (IM C), embora apresente em adolescentes importante variação com a idadee com a maturidade sexual, tem sido considerado como bom indicador de obesidade em adolescentes. No presente estudo foi adotado o termo sobrepeso para se referir tanto a sobrepeso como ao excesso de gordura corporal, assim como tem sido feito por outros autores que definiram em conjunto "sobrepeso e obesidade" em adolescentes com base no IM C ${ }^{10}$ e baixo peso quando o IMC estiver menor que 18,50.

Vários estudos evidenciaram que o problema do sobrepeso/obesidade nos brasileiros adultos 
afeta, proporcionalmente, quase o dobro de muIheres em relação aos homens, de certa forma repetindo o que ocorria com o baixo peso, pois em $1974 / 1975$ o baixo peso atingia 6,8\% dos homense $10,4 \%$ das mulheres, e mesmo tendo-se reduzido esses percentuais (3,4\% e6,1\% em 1997), a diferença entre os sexos foi mantida ${ }^{11}$.

A saúde bucal está atrelada à saúde geral do organismo, assim são necessárias pesquisas para a verificação da prevalência de cárie em adolescentes e relacioná-la com seu estado nutricional para que sejam realizados trabalhos de educação geral ebucal, e orientação dietética nessa população específica.

A obesidade, as doenças dela decorrentes e também a cárie dentária têm no hábito alimentar um importante componente etiológico comum. A OM S ${ }^{12}$ demonstrou em seus estudos que as condições de saúde são afetadas pela obesidade e sobrepeso na infância e na adolescência, sendo dos distúrbios de ordem psicossocial os mais prevalentes etambém a persistência da obesidade na vida adulta.

A American Dietetic Association defendea re lação sinérgica que há entre nutrição e saúde bu$\mathrm{cal}^{13}$, o que reforça a importância da atuação multiprofissional na promoção da saúde, pre venção eintervenção de doenças.

Está ocorrendo uma mudança nos hábitos alimentares dos brasileiros, que vêm substituindo com maior frequência o consumo de alimentos ricos em fibras e nutrientes por alimentos industrializados ricos em gordura e carboidratos, facilitando a instalação da doença, mesmo que os indivíduos estejam expostos a fatores de prote ção amplamente difundidos como os fluoretos ${ }^{14}$.

O importante papel dos açúcares na etiologia da cárie dentária, como a relação entre a condição dentária, a função mastigatória e a ingestão de alimentos, tem sido amplamente demonstrado na literatura científica ${ }^{14}$, acrescentando-se 0 pequeno intervalo de tempo de ingestão dos alimentos e a prática de atividades recreativas em domicílio (assistir televisão, jogar videogame e usar computador).

São necessários dados para a compreensão da cinética temporal, geográfica e social da situação nutricional do país esuas mudanças, uma vez quea alimentação ea nutrição podem influenciar na determinação do processo saúde-doença, bem como no processo de saúde-doença bucal.

0 presente estudo teve por objetivo verificar a prevalência de sobrepeso e obesidade em escolares residentes em um município degrande porte na região centro-oeste do estado de São Paulo e identificar os possíveis fatores associados ao estado nutricional avaliado pelo IMC, relacionando-os com o CPOD.

M aterial e método

\section{Aspectos éticos}

Esta pesquisa foi submetida e aprovada pelo Comitê de Ética em Pesquisa da Faculdade de Odontologia de Bauru (FOB) da Universidade de São Paulo (USP). Foram incluídos na amostra somente os alunos cujos pais e/ou responsáveis concordaram com a participação do menor no estudo.

\section{Amostra}

0 delineamento deste estudo foi exploratório e transversal, envolvendo adolescentes de ambos os gêneros, na idade de 12 anos, que estavam regularmente matriculados em escolas públicas e particulares do município de Bauru, São Paulo ( $n=8)$, distribuídos geograficamente entre as diferentes regiões do município em estudo, com características socioeconômicas peculiares.

0 tamanho da amostra foi calculado utilizando-se dados do SB2000, sendo necessárias 248 unidades amostrais. Foram selecionados 380 adolescentes de forma randomizada, sendo que desses somente 207 trouxeram o Termo de Consentimento Livreeesclarecido assinado pelosresponsáveis e estavam presentes na escola no dia do exame.

\section{Delineamento experimental}

O diagnóstico consistiu em avaliação antropométrica deadolescentes em oito escolas. Antes da avaliação antropométrica, os profissionais envolvidos participaram de treinamento para padronização das medidas, com o objetivo de obter maior precisão dos dados coletados.

Para a detecção do sobrepeso e da obesidade (variáveis dependentes), utilizou-se o Índice de M assa Corporal (IMC; $\mathrm{kg} / \mathrm{m}^{2}$ ) e a correlação com os dados antropométricos obtidos por meio da aferição do peso e estatura em balança específica (antropométrica) através da fórmula15:

$$
I M C=\frac{\text { Peso }}{\text { (Altura } \times \text { Altura) }}
$$

A classificação foi feita de acordo com a Tabela 1. 


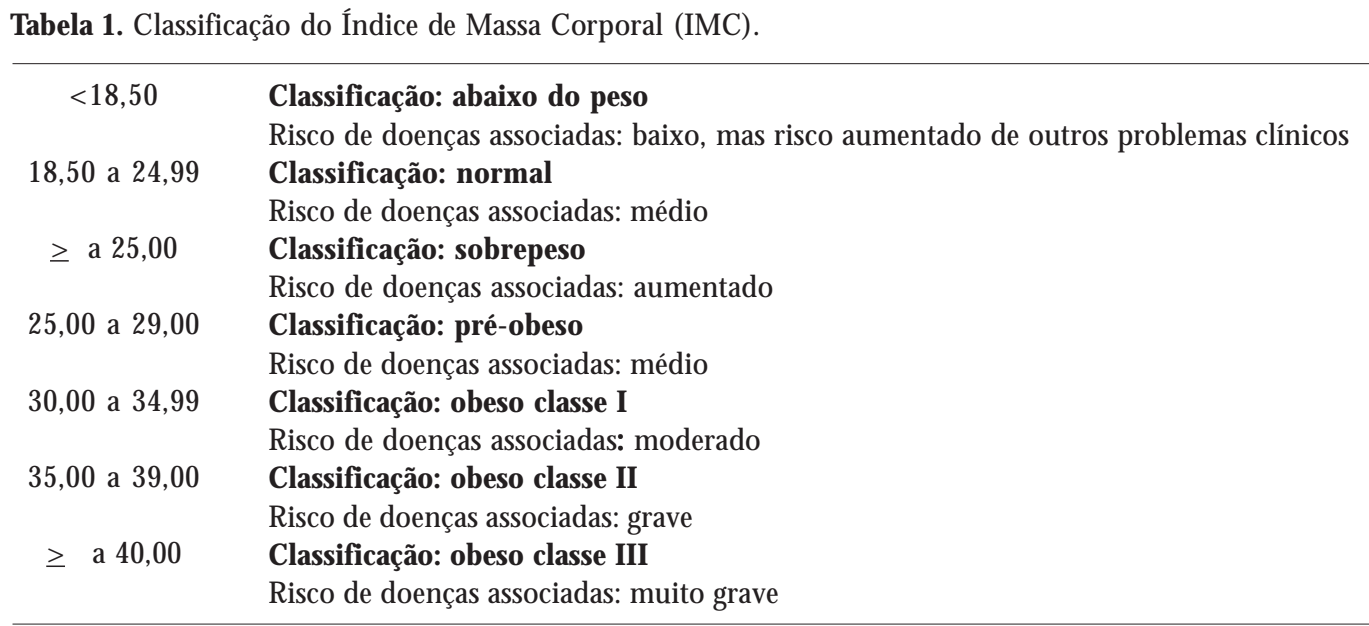

Esse indicador representa a relação entre o peso em quilos ea altura ao quadrado em metros. Pessoas com IMC abaixo de 18,5 quilos/metro quadrado são consideradas como portadoras de déficit de peso. 0 excesso de peso écaracterizado pelo I M C igual ou superior a 25 quilos por metro quadrado, ea obesidade por índiceigual ou supe rior a 30 quilos por metro quadrado.

As medidas antropométricas foram realizadas em todos os participantes da pesquisa. Todos os adolescentes foram pesados vestindo ape nas roupas leves, sem portar objetos pesados, em posição ortostática, como os pés juntos, sem calçado, olhando para a frente, como preconiza Anjos ${ }^{16}$, no centro da balança, com os braços esticados ao lado do corpo esem se movimentar. A leitura da estatura foi feita no milímetro mais próximo ${ }^{9}$, com os pés e calcanhares paralelos, ombros e nádegas encostados na balança. A balança foi colocada em superfícies lisas para se evitarem oscilações nas medidas.

A coleta dos dados consistiu na tomada das medidas de peso, estatura e um questionário de autorresposta composto de: identificação, quantidadeefrequência de consumo usual de alimentos (lista com 73 alimentos), hábitos alimentares, características antropométricas familiares e atividadefísica.

Os adolescentes foram divididos em grupos segundo as medidas de peso: $\mathrm{Gl}$ l=obesos, $\mathrm{G} 2=$ pré-obesos, $\mathrm{G} 3=$ =baixo peso e $\mathrm{G} 4=$ peso normal, nos dois tipos de escolas.

Quanto aos problemas bucais, os participantes do estudo foram examinados segundo o critério do SB Brasil ${ }^{17}$ eno dia do exame receberam um fôlder explicativo sobrehigiene bucal eexplicações individuais sobre saúde bucal e levaram 0 registro da avaliação antropométrica, o qual foi entregue aos pais.

Foi realizada a calibração de dois examinadores, em queo teste deKappa revelou uma concordância de $94,71 \%(k=0,90)$ intraexaminador e $93,27 \%$ ( $k=0,86 \%)$ interexaminador para as condições da coroa dentária. Os instrumentos utilizados para o exame intrabucal foram: espelho bucal plano $n=5$, espátulas de madeira descartáveis e sonda da OMS (sonda CPI - ball point) para levantamentos epidemiológicos embaladas em papel grau cirúrgico e autoclavadas, e a ficha de exame elaborada para a pesquisa quetem como base a ficha do SB Brasi ${ }^{18}$. Quanto ao cálculo de peso corporal, contou-se com a aferição em balança antropométrica para peso e altura e aplicação do cál culo do IMC e questionário individual sobre atividade física e características familiares.

Os adolescentes foram examinados em cadeiras escolares comuns e próximas à janela existente, sendo as cortinas total mente abertas, com luz natural (ambiente), salientando-se que não houve escovação prévia ou profilaxia e secagem dos dentes previamente ao exame e que ele foi desenvolvido antes do intervalo para a merenda escolar. Realizou-se avaliação da correlação entre a prevalência de cárie dentária e os diferentes grupos de massa corpórea.

Empregou-se também um questionário estruturado autoaplicativo, de frequência de consumo alimentar, para este estudo com base no questionário proposto por Pinto ${ }^{18}$ e em questionário utilizado pelo Instituto de M edicina Social 
daUniversidade do Estado do Rio de Janeiro para a área de nutrição.

Quanto ao cálculo de peso corporal, contouse com a aferição em balança antropométrica para peso ealtura e aplicação do cálculo do IMC equestionário individual sobre atividade física e características familiares.

Os dados foram processados através do programa Statistic for Windows 5.1 (Stat Soft Inc), tendo como nível de significância 0,05. Foram utilizados os testes Qui-quadrado e Exato de Fisher. Para verificar a associação entre os eventos, foi adotado o Odds Ratio.

\section{Resultados}

Em relação ao peso corpóreo, 55,93\% apresentaram normal, 35,59\% baixo peso e $8,47 \%$ préobesos, nas escolas particulares. Nas escolas públicas, 52,03\% apresentaram normal, baixo peso $41,22 \%$, pré-obesos $4,73 \%$ e obesos $2,03 \%$; não houvediferençasignificativa $(p=0,45)$ (Gráfico 1 ).

Verificou-se que o CPOD nas escolas públicas foi 2,16 e nas particulares $0,23(p<0,05)$, sendo que $39,2 \%$ das crianças estavam livres de cárie nas escolas municipais e nas particulares, $88,1 \%$ (gráficos 2 e 3 ).

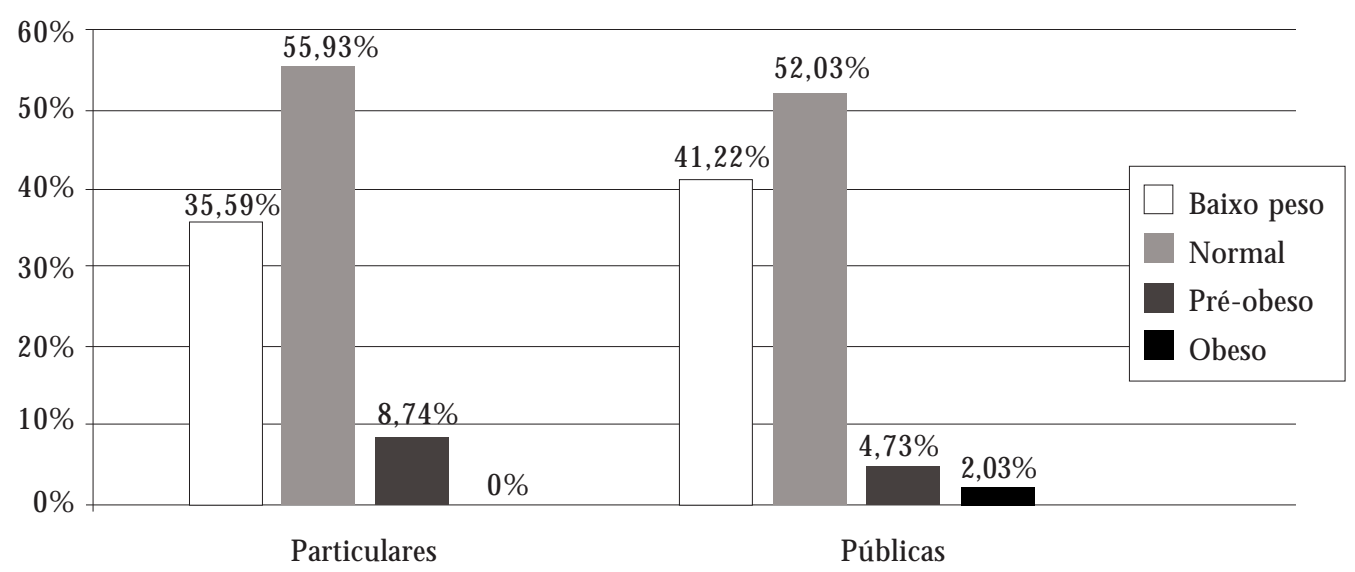

Gráfico 1. Percentual das diferentes classificações de peso, distribuídas nas escolas públicas e particulares.

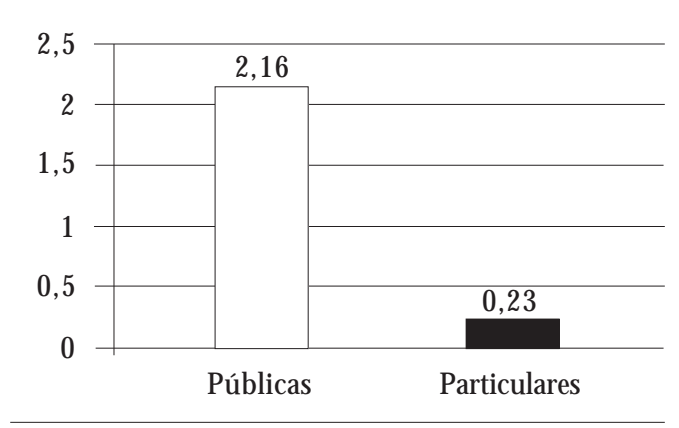

Gráfico 2. Valor do CPOD aos 12 anos, nas escolas públicas e particulares.

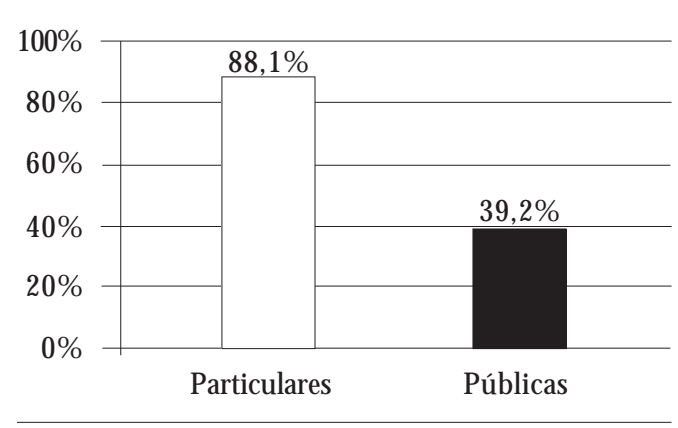

Gráfico 3. Percentual de adolescentes livres de cárie, distribuídos nas escolas públicas e particulares. 
Não houve correlação do maior IMC com 0 incremento de CPOD e constatou-se que $39,61 \%$ dos sujeitos da pesquisa foram classificados no $\mathrm{G} 3$ abaixo do peso e $7 \%$ foram classificados em $\mathrm{G} 1$ ou G2. Houve correlação negativa entre as condições socioeconômicas e o índice de cárie dentária.

\section{Discussão}

0 perfil nutricional das populações tem sofrido modificações, com significativo aumento da obesidade tanto em países desenvolvidos quanto em países em desenvolvimento, sendo considerada como "a epidemia do século XXI". Além disso, por ter forte impacto na morbidade e estar associada às doenças cardiovasculares, diabetes tipo 2 , hipertensão, osteoartrite, entre outras, a obe sidade representa um considerável problema de saúde pública e ainda de grande interesse para a odontologia, visto que ela predispõe o indivíduo a problemas bucais tais como periodontite e cárie dentária, além de erosão, hipersensibilidade dentária e xerostomia, relacionadas ao refluxo gastroesofágico, inclusive em indivíduos submetidos à cirurgia bariátrica'19-21.

Tendo em vista o aumento na prevalência da obesidade, torna-senecessária a avaliação eatenção integral à saúdee do acompanhamento, para melhorar qualidade de vida dos indivíduos.

Os índices antropométricos em adolescentes servem para avaliar o seu crescimento e, indiretamente, para caracterizar o estado nutricional da população $0^{16}$. Em estudos populacionais e na prática clínica, a antropometria constitui-se em importante método diagnóstico, fornecendo estimativa da prevalência e gravidade de alterações nutricionais ${ }^{12}$. A avaliação antropométrica, mesmo quando restrita ao peso e estatura, assume grande importância no diagnóstico nutricional da criança. Isto se deve à sua facilidade de realização, objetividade da medida e possi bilidade de comparação com um padrão de referência de manuseio relativamentesimples, principalmente em estudos populacionais $5^{6,22}$.

A pesar do inegável papel da herança genética no desenvolvimento físico, sabe-se que os fatores ambientais, assim como nutrição, renda familiar e saneamento básico, exercem grande influência no crescimento e desenvolvimento do indivíduo. Grupos de crianças de qualquer parte do mundo crescem no mesmo ritmo se essas condições ideais forem satisfeitas ${ }^{16,23}$.

Um dos mais relevantesfocos de medidas deve ser a prevenção do déficit estatural na população infantil, considerado como produto da fomecrônica, da desigual dade social eda miséria brasileiras $^{24}$. Este corresponde a uma das características mais significativas da desnutrição $0^{25}$ e representa o efeito cumulativo do estresse nutricional sobre o crescimento esquelético. Continua a ser um dos problemas importantes de saúde pública, em razão das consequências desastrosas para o crescimento, o desenvolvimento e até para a sobrevivência de crianças e adolescentes.

Nos países em desenvolvimento, onde a preocupação maior sempre foi a desnutrição, a obesidade começa a ter lugar de destaque, distribuindose por todas as classes sociais. Segundo a PNSN (INAN ${ }^{26}$ ), existem no Brasil $32 \%$ de pessoas com algum grau de excesso de peso. Em relação ao aspecto da distribuição social, as crianças que pertencem ao terço superior derenda das famílias brasileiras apresentam curvas de crescimento que se superpõem aos valores do padrão internacional ${ }^{27,28}$.

A renda familiar tem sido considerada como um fator modulador importante para déficit de altura e obesidade. Há uma tendência superior para déficit de altura com a diminuição da renda familiar per capita. Na direção oposta, há prevalência de aumento da obesidade com o aumento da renda.

$A$ adolescência éum período da vida em que ocorrem grandes mudanças físicas e psicológicas, altamente influenciadas por fatores genéticos, étni cos e ainda pelas diferentes situações sociais e ambientais. Destaca-se a importância da implementação deatividades preventivas deproblemas desaúdee nutrição específicas dentro das políticas de saúde pública, não só para a sua saúde presente como também para a futura ${ }^{29}$.

Goran ${ }^{30}$, em 2001, destacou a necessidade de estudos enfocando as mudanças metabólicas, físicas e comportamentais que ocorrem na adolescência, pois as repercussões dessas mudanças sobre o estado nutricional ea saúde geral podem persistir ao longo da vida. A pesar de estar ocorrendo incremento da obesidade no Brasil ${ }^{9}$, este estudo mostrou baixa prevalência em adolescentes de 12 anos de idade em Bauru. Em adicional, não foi observada diferença estatisticamente significante entre os gêneros; segundo o indicador IMC, os meninos apresentam uma tendência a ter maior comprometimento de peso (\%) do que as meninas (\%) (Tabela 1). Esses achados estão de acordo com os do estudo de Saito ${ }^{23}$, que verificou maior prevalência de desnutrição nos adolescentes do gênero masculino.

$M$ althus-Vliegen et al. ${ }^{31}$ realizaram uma revisão sobre a obesidade e identificaram que este 
problematêm predominância considerável de 15$30 \%$ entre populações europeias e americanas. É uma doença crônica incurável com mortalidade e comorbidade consideráveis. A comorbidade pode ser reduzida substancialmente por perda de peso moderada de $5-15 \%$. A causa principal da obesidade é um desequilíbrio entre a entrada da energia e a despesa de energia. Consequentemente, o tratamento começa com uma dieta restrita da energia, uma redução do estilo de vida sedentário, atividade física aumentada e terapia comportável de mudar hábitos alimentares. Quando necessário, esse tratamento pode ser seguido pela farmacoterapia ou pela cirurgia. A obesidade é relacionada a diversos aspectos da saúde oral, tais como cárie, periodontites e xerostomia. Os autores citados destacaram também que a obesidade pode ter implicações para a realização do tratamento odontológico.

A obesidade, as doenças dela decorrentes e a cárie dentária têm no hábito alimentar um importante componenteetiológico comum. Asconsequências da obesidade para a saúde são muitas, variando desde maior risco de morte prematura até diversos problemas não fatais, mas debilitadores da qualidade de vida dos indivíduos. Os hábitos alimentares relacionados à obesidade podem também determinar maior prevalência de cárie dentária, já que tanto a quantidade de sacarose ingerida quanto a frequência de ingestão são importantes fatores envolvidos em sua etiologia. Os profissionais de saúde devem auxiliar e conscientizar os pais sobre os efeitos imediatos dos hábitos alimentares inadequados sobre a saúde da criança e do adolescente, principalmente em relação à obesidade e à cárie dentária ${ }^{14}$.

Com o objetivo de investigar a associação entre prevalência de cárie e o índice de massa corpórea em escolares alemães, foram avaliados 1.290 alunos (648 meninos, 642 meninas). Os resultados demonstraram que $3,6 \%$ das crianças eram desnutridas, $74,8 \%$ tinham peso normal, $11,9 \%$ sobrepeso e $9,7 \%$ eram obesas. As crianças desnutridas mostraram os dentes saudáveis em $44,7 \%$; aqueles com peso normal em $40,7 \%$, visto que as crianças com sobrepeso eas crianças obesas mostraram os dentes saudáveis em 30,5\% e $31,7 \%$, respectivamente. Este estudo demonstrou uma associação significativa entre a frequência da cárie e o peso nos alunos ${ }^{32}$.

Um estudo conduzido por Marshall et al. ${ }^{33}$ com crianças americanas teve por objetivo determinar se havia associação entre prevalência de cárie dentária e obesidade. Caso fosse identifica$\mathrm{da}$, a associação relacionaria a dieta e o status so- cioeconômico como fatores de risco adicionais. Os resultados demonstraram que as crianças de baixa classe socioeconômica apresentam cárie dentária e obesidade. Ante esse achado foi proposta a implementação de políticas públicas de saúde para melhorar a educação em relação aos hábitos alimentares para que possa ocorrer a redução do risco de ambas as doenças. Os achados deste estudo vão ao encontro dos de $M$ arshall et al. ${ }^{33}$, uma vez que os adolescentes das escolas públicas apresentaram o CPOD mais elevado e 0 menor percentual de livres de cárie (Gráfico 2).

Adolescentes e crianças foram avaliados em relação à ocorrência de cárie e possíveis fatores etiológicos. Alm ${ }^{34}$ demonstrou que os adolescentes com sobrepeso eobesidadetiveram mais chance de desenvolver cáries proximais quando comparados aos de peso normal. Em adicional, mostrou que o consumo de salgadinhos tipo snack esteve associado à ocorrência de cárie proximal.

Os resultados deste estudo sugerem que 0 profissional de saúde necessita, além de dar orientações sobre alimentação saudável a todos os pacientes, oferecer atenção especial às famílias com as características que demonstraram associação ao sobrepeso. Em nível coletivo, parece importante que a promoção de saúde englobe aspectos educacionais e ambientais tanto para adultos como para crianças, incentivando dieta adequada e atividade física.

Outros estudos relacionados à obesidade e problemas nutricionais com a cárie dentária devem ser desenvolvidos, para que se possam fazer mai ores inferências. Contudo, o el evado consumo de açúcar por adolescentes obesos pode ser considerado um fator de risco para a ocorrência de cárie dentária.

0 conhecimento da tendência das prevalências do sobrepeso, da obesidade e desnutrição na adolescência poderá contribuir para melhor enten dimento do problema esua associação com cárie dentária mesmo em seus estágios iniciais. Poderá também propiciar informações para o desenvolvimento do trabalho em equipe multiprofissional, além de permitir quea saúde pública concentre seus esforços nos grupos de risco ou grupos que sejam mais vulneráveis. Como propõem Pinto et al. ${ }^{32}$, programas preventivos devem ressaltar a importância da nutrição em relação às doen ças gerais e enfatizar os riscos em relação às doenças bucais. Por essa razão, a promoção da alimentação saudável constitui-se numa das estratégias de saúde pública de vital importância para 0 enfrentamento dos problemasalimentares enutricionais ${ }^{27}$. As políticas pú- 
blicas devem englobar uma abordagem integral capaz de prevenir ao mesmo tempo as doenças causadas por deficiências nutricionais, por meio do aumento da resistência orgânica às doenças infecciosas e outras doenças crônicas não transmissíveis associadas, como por exemplo, a redução da obesidade ${ }^{35}$. 0 planejamento de políticas públicas de saúde deve estar pautado no conhe cimento dos problemas e necessidades da população, podendo assim direcionar melhor os recursos disponíveis.

\section{Colaboradores}

SH C Sales-Peres, S Goya, RM F Sant'Anna, HM Silva, AC Sales-Peres, RPR Silva, JRP Laurise J RM Bastos participaram da concepção do trabalho, da análise e interpretação dos dados, de sua redação, da revisão crítica e da leitura e aprovação da versão final.

\section{Agradecimentos}

Agradecemos a colaboração das diretoras e professoras das Escolas Criarte, Batista e Seta de Bauru, da Secretaria M unicipal de Educação, das diretoras, coordenadoras e professoras das escolas municipais, das funcionárias do Departamento de Saúde Coletiva (Sílvia, Rosa e M arta). $E$ à equipe que muito nos auxiliou nesta pesquisa, composta por Fernanda M enechio Costa, Ana Carolina Zampronio Bassi e M aurício Leonardo Magini Rocha. Nosso muito obrigado a todos os adolescentes queanonimamente participaram da pesquisa e aos pais que souberam da importância de cumprir seu papel de cidadania.

\section{Conclusões}

Pode-seconcluir quea cárie dentária e a obesidade são doenças multifatoriais relacionadas aos hábitos alimentares. Os riscos dessas doenças devem ser identificados o mais precocemente possível, para que medidas preventivas e estraté gias possam ser adotadas para reduzir ou minimizar essas ocorrências. A associação entre cárie dentária, obesidade e consumo de açúcar deve ser mais bem estudada para que se possam fazer outras inferências. 


\section{Referências}

1. Vasconcelos VL, Silva GAP. Prevalência de sobrepeso e obesidade em adolescentes masculinos no Nordeste do Brasil, 1980-2000. Cad Saude Publica 2003; 19(5):1445-1451.

2. Standing Committee on Nutrition. Diet-related chronic diseases and double burden of malnutrition in West Africa 2006. London: United Nations System; 2006. (Standing Committee on Nutrition N ews, 33).

3. Uauy R, Albala C, Kain J. Obesity trends in Latin America: transiting from under-to overweight. Journal of Nutrition 2001; 131:893-899.

4. Coutinho JG, Gentil PC, Toral N. A desnutrição e a obesidade no Brasil: o enfrentamento com base na agenda única da nutrição. Cad Saude Publica 2008; 24(2):332-340.

5. Moraes AS, Rosas JB, M ondin L, Freitas ICM. Prevalência de sobrepeso e obesidade e fatores associados em escolares de área urbana de Chilpancingo, Guerrero, M éxico, 2004. Cad Saude Publica 2006; 22(6):1289-1301.

6. World Health Organization (WHO). Physical status: the use and interpretation of anthropometry. Gene va: WHO; 1995. (Technical Report Series 854).

7. Fundação Instituto Brasileiro de Geografia e Estatística (IBGE). Pesquisa Nacional por Amostra de Domicílio; 1990. [acessado 2006 dez 20]. Disponível em: http://www.ibge.gov.br/home/estatistica/ populacao/condicaodevida/pof/2002analise/ pof2002analise.pdf

8. Guo S, Cameron W. Tracking of body mass index in children in relation to overweight in adulthood. American Journal of Clinical Nutrition 1999; 70(Suppl.):145S-148S.

9. Silva CEF, Mattos LES, Gonçalves-Silva RM V, Le mos-Santos M G, Botelho C. Estado nutricional de alunos de 7 a 9,9 anos da Escola Estadual Mário de Castro, do Bairro Pedra 90 - Cuiabá - Mato Grosso. Cad Saude Publica 2000; 1:77-95.

10. Himes JH, Dietz WH. Guidelines for overweight in adolescent preventive services: recommendations from an expert committee. American Journal of Clinical Nutrition 1994; 59:307-316.

11. Burlandy L, Anjos LA. Acesso a vale-refeição e estado nutricional de adultos beneficiários do Programa de Alimentação do Trabalhador no Nordeste e Sudeste do Brasil, 1997. Cad Saude Publica 2001; 17:1457-1464.

12. World Health Organization) (WHO). Working Group on Purpose, Use and Interpretation of Anthropometric Indicators of Nutritional Status: use and interpretation of anthropometric indicators of nutritional status. Bulletin of the World Health Organization 1986; 64(6):929-941.

13. American Dental Association (ADA). Position of the American Dietetic Association: oral health and nutrition. Journal of the American Dietetic Association 2007; 107:1418-1428.

14. Traebert J, Moreira EAM, Bosco VL, Almeida, ICS Transição alimentar: problema comum à obesidade e à cárie dentária. Revista de Nutrição 2004; $17(2): 247-253$
15. Must A, Dallal GE, Dietz WH. Reference data for obesity: 85th and 95th percentiles for body mass index (wt/ht2) and triceps skinfold thicknes. American Journal of Clinical Nutrition 1991; 53:839-846.

16. Anjos L. Antropometria nutricional: uso de dados de peso e de altura na avaliação do estado nutricional de crianças com menos de 10 anos de idade. Revista Ciência e M ovimento 1988; 2(1):7-16.

17. Brasil. M inistério da Saúde. Secretaria de Políticas de Saúde. Departamento de Atenção Básica. Área Técnica de Saúde Bucal. Projeto SB Brasil: condições de saúde bucal da população brasileira - resultados principais. Brasília: M inistério da Saúde; 2004.

18. Pinto VG. Saúde bucal coletiva. São Paulo: Editora Santos; 2008.

19. Oliveira SS, Santos IS, Silva JFP, Machado EC. A prevalência e fatores associados à doença do refluxo gastroesofágico. Arquivos de Gastroenterologia 2005; 42(2):116-121.

20. Heling I, Sgan-Cohen HD, Itzhaki M, Beglaibter N, Avrutis O, Gimmon Z. Dental complications following gastric restrictive bariatric surgery. 0 besity Surgery 2006; 16(9):1131-1134.

21. Barron RP, Carmichael RP, Marcon MA, Sàndor GK. Dental erosion in gastroesophageal reflux disease. Journal of Canadian Dental Association 2003; 69(2):84-89.

22. Douek PC, Leone C. Nutritional status of young children: a comparison among three anthropometric classifications. Journal of Pediatrics 1995; 71(3):139-144.

23. Saito MI. Avaliação nutricional na adolescência: a escolha do referencial. Journal of Pediatrics 1993; 69:165-175.

24. Guigliani ERJ. Baixa estatura: um mal da sociedade brasileira. Journal of Pediatrics 1994; 70:261-262.

25. Laurentino GEC, Arruda IKG, Arruda BKG. Nanismo nutricional em escolares no Brasil. Revista Brasileira Saúde M aterno Infantil 2003; 3:377-385.

26. Instituto Nacional de Alimentação e Nutrição. Pesquisa nacional sobre saúde e nutrição: condições nutricionais da população brasileira: adultos e idosos. Brasília: INAN; 1991.

27. Batista-Filho $M$, Rissin $A$. A transição nutricional no Brasil: tendências regionais e temporais. Cad Saude Publica 2003; 19(Sup.1):181-191.

28. Monteiro CA, Conde WL, Castro IRR. A tendência cambiante da relação entre escolaridade e risco de obesidade no Brasil (1975-1997). Cad Saude Publica 2003; 19(Supl.1):S67-S75.

29. M agalhães VC, Azevedo G, Mendonça S. Prevalência e fatores associados a sobrepeso e obesidade em adolescentes de 15 a 19 anos das regiões Nordeste e Sudeste do Brasil, 1996 a 1997. Cad Saude Publica 2003; 19(Supl.1):S129-S139.

30. Goran MI. M etabolic precursors and effects of obesity in children: a decade of progress, 1990-1999. American Journal of Clinical Nutrition 2001; 73(2):158-171.

31. Mathus-Vliegen EM, Nikkel D, Brand HS. Oral aspects of obesity. International Dental Journal 2007; 57(4):249-256. Review. 
32. Pinto A, Kim S, Wadenya R, Rosenberg $\mathrm{H}$. Is there an association between weight and dental caries among pediatric patients in an urban dental school? A correlation study. Journal of Dental Education 2007; 71(11):1435-1440.

33. Marshall TA, Eichenberger-Gilmore JM, Broffitt BA, Warren JJ, Levy SM. Dental caries and childhood obesity: roles of diet and socioeconomic status. Community Dentistry and Oral Epidemiology 2007; 35(6):449-458.

34. Alm A. On dental caries and caries-related factors in children and teenagers. Swedish Dental Journal Suppl 2008; 195:7-63.

35. Brasil. M inistério da Saúde. Secretaria de Atenção à Saúde. Departamento de Atenção Básica. Coordenação-Geral da Política de Alimentação e Nutrição. Guia alimentar para a população brasileira: promovendo a alimentação saudável. Brasília: Ministério da Saúde; 2006.

Artigo apresentado em 13/08/2007

Artigo aprovado em 20/08/2008

Versão final em 17/09/2008 